\title{
PERFIL DE ELEMENTOS INORGÂNICOS EM AMOSTRAS DE MEL DO ESTADO DO MARANHÃO COMO UM POTENCIAL BIOINDICADOR DE ORIGEM GEOGRÁFICA
}

\author{
Sonaly S. Leal ${ }^{\mathrm{a}, *, \odot, ~ E l i a n e ~ R . ~ S o u s a ~}{ }^{\mathrm{b}}$, Gilmar Silvério ${ }^{\mathrm{a}}$ e Paulo C. Lima ${ }^{\mathrm{a}}$ \\ ${ }^{a}$ Departamento de Química, Instituto Federal de Educação, Ciência e Tecnologia do Maranhão - Campus Monte Castelo 65030-005, \\ São Luís - MA, Brasil

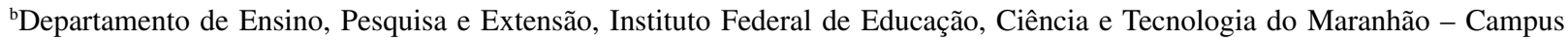 \\ Maracanã, 65095-460, São Luís - MA, Brasil
}

Recebido em 17/07/2021; aceito em 30/11/2021; publicado na web em 27/01/2022

\begin{abstract}
PROFILE OF INORGANIC ELEMENTS IN HONEY SAMPLES FROM THE MARANHÃO STATE AS A BIOINDICATOR OF GEOGRAPHICAL ORIGIN. Honey is a substance that contains a variety of macro and micro minerals present in the range between $0.02-1.03 \%$. The main objective of this study was to determine the concentration of elements inorganic in 15 honey samples collected in various regions of Maranhão (Northeast Brazil), using highly sensitive analytical techniques, such as inductively coupled plasma optical emission spectrometry (ICP-OES), in addition to applying multivariate statistical tools, such as Principal Component Analysis (PCA) and Hierarchical Cluster Analysis (HCA) in the treatment of data. The method presented detection limits between 0.4 to $2.8 \mathrm{mg} \mathrm{kg}^{-1}$. Fifteen samples and 19 metals were analyzed, and two of these samples had high levels of lead, exceeding the limits allowed by Brazilian legislation. Thus, it can be concluded that the presence of potentially toxic metals in honey is an indication that the production areas may be contaminated by different external sources. The chemometric analysis of the data by PCA and HCA allowed to classify honey in two main groups: honey produced in urban areas and honey produced in rural areas with PCA explaining $73 \%$ of the data variance with the sum of the first 3 components.
\end{abstract}

Keywords: honey; chemometry; bioindicator; ICP-OES.

\section{INTRODUÇÃO}

O mel é uma substância viscosa produzida pelas abelhas, composto por nutrientes essenciais ao organismo que vão desde aminoácidos, carboidratos, açúcares, proteínas, água e minerais. Apesar da grande diversidade de espécies de abelhas produtoras de mel próprio para o consumo, a Apis mellifera, também conhecida popularmente como abelha africanizada se destaca por ser altamente produtiva e ativa o ano inteiro. ${ }^{1} \mathrm{~A}$ composição inorgânica presente no mel, representa cerca de $0,1-1 \%$, podendo variar de acordo com sua origem botânica e geográfica. Os teores desses elementos são influenciados diretamente por uma série de fatores como poluição industrial, procedimentos incorretos durante a produção do mel, além de solos contaminados por pesticidas e fertilizantes contendo elementos contaminantes no entorno das colmeias. ${ }^{2}$

Com o crescimento da atividade apícola no estado do Maranhão, existe uma preocupação em relação à comercialização do mel na forma in natura, tendo em vista que a longo prazo essa prática pode ser prejudicial à saúde, em razão da possibilidade de contaminantes em sua composição. Durante a fabricação do mel, é comum ocorrerem alterações de diversas origens. Elementos como $\mathrm{Cd}, \mathrm{Pb}, \mathrm{Cu}, \mathrm{Zn}, \mathrm{Mn}$, $\mathrm{Ni}$ e $\mathrm{Sr}$ podem ser os principais responsáveis pela contaminação de produtos apícolas, visto que estando em níveis acima do permitido pela legislação, representam ameaças ao bem-estar humano em virtude de seus efeitos acumulativos e sua toxicidade no organismo, já que não podem ser degradados. ${ }^{3}$

A contaminação por esses metais está ligada diretamente ao ambiente em que este é produzido por meio do comportamento do sistema solo-planta, no qual os metais são transportados pela raiz, entram em contato com o néctar, até chegarem as etapas de produção das abelhas. A grande variedade de ambientes também implica nas

*e-mail: sonaly.leal15@hotmail.com diferentes concentrações dos metais nos méis de cada lugar. ${ }^{4,5}$ Assim, o teor de metal no mel pode ser uma característica nutritiva positiva, bem como um indicativo de contaminação do local onde é produzido. ${ }^{6}$ Alguns estudos sugerem que o mel pode ser útil como indicador de poluição ambiental por elementos inorgânicos, como $\mathrm{As}, \mathrm{Cd}, \mathrm{Pb}$ e $\mathrm{Hg}$, pois as abelhas podem estar expostas a contaminantes em uma área de aproximadamente $7 \mathrm{~km}^{2}$ ao redor do apiário. ${ }^{1}$

Em decorrência disso, o mel, por possuir propriedades físicoquímicas que variam conforme a sua origem botânica e geográfica, revela sua funcionalidade como bioindicador, onde sua composição permite que sejam averiguados e monitorados os locais em que são produzidos. ${ }^{7}$ Como forma de identificar elementos contaminantes no mel, foi feito o uso da técnica de Espectrometria de Emissão Óptica com Plasma Indutivamente Acoplado (ICP OES). Para obter uma melhor avaliação do conteúdo de elementos inorgânicos em um grupo de amostras, ferramentas quimiométricas têm sido usadas para identificar um modelo multivariado de agrupamento, usando as variáveis organizadas em uma matriz de dados. ${ }^{8}$ As duas técnicas de análise multivariada mais conhecidas são as Análises Hierárquica de Clusters (HCA) e a Análise de Componentes Principais (PCA). A PCA e a HCA são metodologias exploratórias que visam evidenciar similaridades ou diferenças entre amostras em um determinado conjunto de dados. ${ }^{9}$

A determinação da concentração de elementos inorgânicos em mel tem sido reportada por estudos com amostras de diferentes locais, cada um apresentando características únicas que permitem compreender a presença de altos ou baixos níveis de cada elemento em sua composição. Silveira-Júnior et al., relataram uma variedade botânica de pólen usados como indicadores de mel no nordeste do Brasil, determinando elementos como $\mathrm{As}, \mathrm{Cd}, \mathrm{Cr}, \mathrm{Pb}$ e $\mathrm{Hg}$ com uso da técnica de FAAS ${ }^{10}$; Dhahir e Hemed ${ }^{11}$ quantificaram metais tóxicos e elementos traço em diferentes amostras de mel do Iraque utilizando as técnicas FAAS e GFAAS; um estudo feito por 
Hungerford et al. ${ }^{12}$ revelou o impacto causado no meio ambiente através da determinação de elementos traços essenciais e elementos majoritários no mel de diferentes regiões da Austrália, mostrando uma diferença significativa entre o teor de mineral do mel da área urbana e o mel da área rural. Velimirovic et al. ${ }^{13}$, investigaram o conteúdo mineral fenólico e atividade antioxidante de amostras de mel na Sérvia, onde foram determinados elementos majoritários como $\mathrm{Na}, \mathrm{Ca}, \mathrm{Mg}$ e K. Elementos tóxicos como $\mathrm{Pb}$ e Cd também foram encontrados em algumas amostras, revelando um possível indicador de poluição ambiental da área estudada. ${ }^{11-13}$ Com relação a este estudo, vale ressaltar que ainda não há na literatura nenhum trabalho com essa temática no estado do Maranhão, localizado na região Nordeste do Brasil.

O presente estudo possui um caráter prospectivo, e pretende encontrar uma correlação entre a composição mineral do mel, sua origem geográfica e o teor de poluição ambiental, utilizando para isso, métodos de classificação, como PCA e HCA, além de verificar algum possível risco toxicológico relacionado a ingestão do mel.

\section{PARTE EXPERIMENTAL}

\section{Coleta das amostras}

Por se tratar de um estudo ainda em prospecção, inicialmente esta pesquisa foi conduzida com base na coleta de 15 amostras de mel, obtidas de produtores rurais de diferentes municípios do estado do Maranhão. A região possui uma vasta costa litorânea e uma flora bastante diversificada, destacando-se os manguezais, campos babaçuais e vegetação restinga. Esses fatores juntamente com o clima tropical predominante contribuem para o desenvolvimento do setor de produção melífera do estado.

Recolhidas no período de janeiro a maio de 2019, todas as amostras foram armazenadas em vidro âmbar, encaminhadas ao laboratório, mantidas em temperatura ambiente e na ausência de luz. Essas amostras foram preparadas para digestão úmida via sistema aberto com aquecimento convencional para posteriores análises de determinação de metais. A Figura 1 mostra a origem das amostras de mel analisadas neste trabalho.

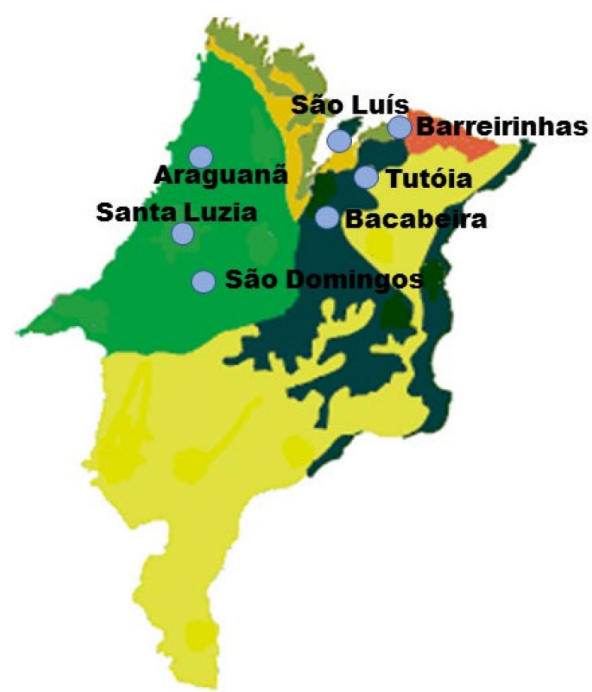

Figura 1. Regiões de coleta de mel do estado do Maranhão.

\section{Preparação das amostras de mel}

Para a mineralização das amostras, o método utilizado foi a decomposição por via úmida em sistema aberto com aquecimento convencional, proposto por Perrielo Leme et al., com algumas adaptações e modificações com relação à massa da amostra e ao volume do ácido utilizado. ${ }^{14}$ As proporções dos reagentes oxidantes foram estabelecidas após algumas análises onde a quantidade de ácido puro utilizado foi eficiente na degradação da matéria orgânica. Foram pesados $0,3 \mathrm{~g}$ de amostra de mel em tubos de vidro borosilicato e adicionados $3 \mathrm{ml}$ de $\mathrm{HNO}_{3}$ (TEDIA) com auxílio de micropipeta. A mistura passou por uma digestão prévia em repouso sob temperatura ambiente, por um período de $15 \mathrm{~h}$. Após esse período, adicionou-se $1 \mathrm{ml}$ de $\mathrm{H}_{2} \mathrm{O}_{2}\left(30 \% \mathrm{~mm}^{-1}\right)$ (Emsure ${ }^{\circledR}$ MERCK) aos frascos e deixouse em repouso por mais $2 \mathrm{~h}$. Então, a mistura foi aquecida a $100{ }^{\circ} \mathrm{C}$ gradativamente em bloco digestor (Tecnal, modelo TE-040/25) por um período de $3 \mathrm{~h}$, e os tubos foram parcialmente tampados com auxílio de vidros de relógio a fim de evitar contaminação cruzada. Ao final do processo, esperou-se a solução arrefecer até a temperatura ambiente, transferiu-se para tubos tipo Falcon estéreis e todas as amostras foram avolumadas para $30 \mathrm{ml}$ com água deionizada (Milli-Q® System Millipore, USA). De maneira semelhante, digestões sem amostras de mel, empregadas como branco também foram submetidas ao mesmo procedimento de digestão. Cada análise foi realizada em triplicata.

\section{Curva de calibração}

Uma curva analítica foi preparada utilizando-se soluções de ácido nítrico 5\%, água deionizada e um padrão Multielementar com certificado SpecSol, do qual foi preparado uma solução-estoque que serviu de base para as soluções-padrão.

A concentração dos elementos nas soluções analíticas variou de 10 a $500 \mu \mathrm{g} \mathrm{L}^{-1}$, sendo a faixa linear para o Fe, Ba e Li (10-100 $\left.\mu \mathrm{g} \mathrm{L}^{-1}\right)$; para o $\mathrm{Cd}, \mathrm{Ni}, \mathrm{Pb}, \mathrm{Ag}, \mathrm{Cu}, \mathrm{Co}, \mathrm{Cr}, \mathrm{Mn}, \mathrm{Zn}, \mathrm{K}, \mathrm{Mg}, \mathrm{Al}, \mathrm{Ga}, \mathrm{In}, \mathrm{Sr}$ (10-250 $\left.\mu \mathrm{g} \mathrm{L}^{-1}\right)$ e para o B $\left(10-500 \mu \mathrm{g} \mathrm{L}^{-1}\right)$. As soluções finais foram avolumadas em balões de 25 e $50 \mathrm{ml}$.

As análises foram realizadas utilizando-se um Espectrômetro de Emissão Óptica com Plasma Indutivamente Acoplado, ICP OES Perkin Elmer, modelo Optima 7000 DV. As configurações de operação (Tabela 1) foram determinadas com base no manual do equipamento e em referências de estudos feitos com análise de ICP OES em matriz de mel. Também foram selecionadas as linhas espectrais referentes aos metais escolhidos para as análises. As linhas espectrais foram pré-estabelecidas para os seguintes metais: Cd $(226.502 \mathrm{~nm}), \mathrm{Ni}(221.648 \mathrm{~nm})$, $\mathrm{Pb}(220.353 \mathrm{~nm}), \mathrm{Cu}(324.752 \mathrm{~nm}), \mathrm{Ag}$ (328.068 nm), Co $(228.616 \mathrm{~nm}), \mathrm{Cr}(267.716 \mathrm{~nm}), \mathrm{Mn}(257.610 \mathrm{~nm}), \mathrm{Zn}(213.857 \mathrm{~nm})$, $\mathrm{K}(766.490 \mathrm{~nm}), \mathrm{Mg}(279,553 \mathrm{~nm}), \mathrm{Fe}(259,939 \mathrm{~nm}), \mathrm{Al}$ (396.153 nm), Ga (417.206 nm), In (325.609 nm), Ba (455.403 nm), $\mathrm{Sr}(407.771 \mathrm{~nm}), \mathrm{Li}(670.784 \mathrm{~nm}), \mathrm{B}(249.677 \mathrm{~nm}) .{ }^{15-16}$

Tabela 1 - Condições de operação do ICP OES

\begin{tabular}{lc}
\hline Parâmetros \\
\hline Fluxo do Plasma & $15 \mathrm{~L} \mathrm{~min}^{-1}$ \\
Fluxo do gás Nebulizador & $0,5 \mathrm{~L} \mathrm{~min}^{-1}$ \\
Fluxo do gás Auxiliar & $0,5 \mathrm{~L} \mathrm{~min}^{-1}$ \\
Fluxo da Amostra & $1,5-2,5 \mathrm{~mL} \mathrm{~min}$ \\
Potência da Fonte de Radiofrequência & $1450 \mathrm{~W}$ \\
\hline
\end{tabular}

\section{Linearidade, limite de detecção e limite de quantificação}

A linearidade de um método é definida como sua capacidade de fornecer um sinal (y) que é diretamente proporcional à concentração do analito (x). Essa relação é expressa pela equação matemática 
denominada curva de calibração. A estimativa dos coeficientes de uma curva de calibração pode ser efetuada a partir do método matemático conhecido regressão linear, onde além dos coeficientes de regressão (a e b), também é possível calcular o coeficiente de correlação (r). ${ }^{17} \mathrm{~A}$ correlação representada pelo modelo matemático entre $\mathrm{x}$ e y é representada pelo coeficiente de correlação $(\mathrm{R})$, como demonstra a equação 1 .

$$
y=a+b x
$$

O limite de detecção (LD) é um parâmetro que representa a menor concentração da substância em análise possível de ser detectada, mas não necessariamente quantificada, utilizando um determinado procedimento experimental. O limite de quantificação (LQ), por outro lado, representa a menor concentração quantificável da substância em estudo. ${ }^{17}$ Os limites de detecção (LD) e quantificação (LQ) foram obtidos através das seguintes equações 2 e 3 :

$$
\begin{gathered}
L D=\frac{3 \times D P}{b} \\
L Q=\frac{10 \times D P}{b}
\end{gathered}
$$

onde: $\mathrm{DP}=$ desvio padrão do branco; $\mathrm{b}=$ coeficiente angular da reta.

\section{Análises estatísticas}

O tratamento estatístico multivariado foi realizado através das ferramentas Análise Hierárquica de Clusters (HCA) e Análise de Componentes Principais (PCA), por meio de um software, o Unscrumbler $X$ versão 10.4, permitindo assim, fazer a classificação das amostras em função de sua origem geográfica e, como consequência, demonstrar um indicativo de poluição ambiental.

Como pré-processamento, a matriz de dados foi auto-escalonada, no que consiste em centrar os dados na média e dividi-los cada um pelo desvio padrão (Equação 4), de modo que todas as variáveis tenham a mesma importância, ou seja, o mesmo peso. ${ }^{18}$

$$
Z_{i j}=\frac{x_{i j}-\bar{X}_{j}}{S_{j}}
$$

onde: $x i j=$ valor para o objeto $\mathrm{i}$ da variável $\mathrm{j} ; \bar{X}_{j}=$ média para variável $\mathrm{j} ; S j=$ desvio padrão para variável $\mathrm{j}$

\section{RESULTADOS E DISCUSSÃO}

A faixa de linearidade estudada ( 10 a $500 \mu \mathrm{g} \mathrm{L}^{-1}$ ) exibiu valores do coeficiente de correlação satisfatórios, variando entre 0,995 - 0,999, como mostrado na Tabela 2. Os elementos como $\mathrm{Cd}, \mathrm{Ag}, \mathrm{Co}, \mathrm{Cr}$ e
Li apresentaram valores abaixo limite de detecção (LD) e limite de quantificação em todas as amostras. Os valores aqui encontrados podem ser comparados com estudos feitos com méis de diferentes origens, como por exemplo, Conti et al. ${ }^{19}$ que fez uso da técnica de ICP OES para a determinação de metais em mel, encontrou valores comparáveis para $\mathrm{Mg}$ (LD-2 mg kg-1), Fe (LD -3 mg kg-1), Al (LD -1 $\left.\mathrm{mg} \mathrm{kg}^{-1}\right)$ e $\mathrm{Li}\left(\mathrm{LD}-1 \mathrm{mg} \mathrm{kg}^{-1}\right)$, por exemplo.

\section{Massa característica dos elementos químicos}

Ainda que não existam valores máximos permitidos na legislação para todos os elementos investigados para a matriz de mel, os resultados apresentados sugestionam o uso do mel como uma proposta de monitoramento da qualidade ambiental. Além disso, pode prospectar uma possível aplicação do mel para estudos de autenticidade de origem geográfica, com base na estimativa do seu conteúdo de elementos inorgânicos.

$\mathrm{Na}$ tabela 3, observa-se que, dentre as amostras analisadas, a amostra "SLZ2" foi a que apresentou mais elementos em sua composição, incluindo alguns potencialmente tóxicos como $\mathrm{Ni}$ e $\mathrm{Pb}$. Os níveis de variação da massa característica dos metais podem ser explicados principalmente por sua origem botânica e geográfica além da influência de fatores antropogênicos.

Quantitativamente, o potássio foi o elemento considerado mais abundante com massas que variaram entre $29,1-1594,7 \mathrm{mg} \mathrm{kg}^{-1}$ (amostras pertencentes aos municípios de Tutóia e Barreirinhas, respectivamente).

Estudos realizados com matriz orgânica de mel em ICP OES relataram valores semelhantes para o potássio: Chudzinska e Baralkiewicz ${ }^{20}$ encontraram $995 \mathrm{mg} \mathrm{kg}^{-1}$; Mendes $^{21}$, relatou valores entre 440,4-1042,3 $\mathrm{mg} \mathrm{kg}^{-1}$. Esses altos níveis de potássio podem ser favorecidos principalmente pela sua facilidade de absorção pela vegetação. ${ }^{17-20} \mathrm{O}$ magnésio foi o segundo elemento mais abundante encontrado nas amostras com massas que variaram entre 3,35 e $113,2 \mathrm{mg} \mathrm{kg}^{-1}$, ambas pertencentes ao município de Barrerinhas. Esses valores estão em semelhança com massas encontradas pelos estudos de Oliveira e Nagashima. ${ }^{22}$ A ocorrência desses macronutrientes nos méis pode ser explicada por vários fatores, dentre eles, o fato de que quando a produção acontece em áreas litorâneas, o mar funciona como uma fonte geogênica de potássio, devido seu transporte através de aerossol marinho, favorecendo principalmente a presença de potássio no mel. ${ }^{23}$

Os valores encontrados para o manganês e zinco foram 0,95-2,74 e 1,0-3,94 $\mathrm{mg} \mathrm{kg}^{-1}$, respectivamente. A presença desses elementos nos meliponários podem ser originados principalmente de atividades antropogênicas ou práticas inadequadas de apicultura. ${ }^{24}$

As massas para o ferro ficaram entre $0,6-18,6 \mathrm{mg} \mathrm{kg}^{-1}$ e foram comparáveis a quantidades obtidas por Yucel e Sultanoglu e Squadrone e colaboradores. ${ }^{25-26}$

Já os elementos potencialmente tóxicos como níquel $(0,9 \mathrm{mg} \mathrm{kg}$ $\left.{ }^{1}\right)$ e chumbo (1,8-3,0 $\left.\mathrm{mg} \mathrm{kg}^{-1}\right)$, que quando em excesso são

Tabela 2 - Determinação de parâmetros analíticos obtidos para os 19 metais analisados por ICP OES em amostras de mel

\begin{tabular}{lcccccccccc}
\hline & $\mathrm{Cd}$ & $\mathrm{Ni}$ & $\mathrm{Pb}$ & $\mathrm{Cu}$ & $\mathrm{Ag}$ & $\mathrm{Co}$ & $\mathrm{Cr}$ & $\mathrm{Mn}$ & $\mathrm{Zn}$ & $\mathrm{K}$ \\
\hline $\mathrm{LD}\left(\mathrm{mg} \mathrm{kg}^{-1}\right)$ & 0,5 & 0,5 & 0,4 & 0,7 & 0,5 & 0,6 & 0,6 & 0,6 & 0,6 & 0,7 \\
$\mathrm{LQ}\left(\mathrm{mg} \mathrm{kg}^{-1}\right)$ & 1,78 & 1,76 & 1,4 & 2,34 & 1,78 & 2,05 & 2,05 & 2,11 & 2,02 & 2,55 \\
$\mathrm{R}^{2}$ & 0,999 & 0,999 & 0,999 & 0,999 & 0,999 & 0,999 & 0,999 & 0,999 & 0,999 & 0,999 \\
\hline & $\mathrm{Mg}$ & $\mathrm{Fe}$ & $\mathrm{Al}$ & $\mathrm{Ga}$ & $\mathrm{In}$ & $\mathrm{Ba}$ & $\mathrm{Sr}$ & $\mathrm{Li}$ & $\mathrm{B}$ & \\
\hline $\mathrm{LD}\left(\mathrm{mg} \mathrm{kg}^{-1}\right)$ & 1,1 & 0,5 & 0,8 & 2,0 & 2,2 & 0,5 & 0,4 & 0,5 & 2,8 & \\
$\mathrm{LQ}\left(\mathrm{mg} \mathrm{kg}^{-1}\right)$ & 3,72 & 1,9 & 2,77 & 6,6 & 7,58 & 1,76 & 1,33 & 1,8 & 9,6 & 0,9 \\
$\mathrm{R}^{2}$ & 0,998 & 0,998 & 0,999 & 0,995 & 0,996 & 0,998 & 0,999 & 0,998 & 0,997 & \\
\hline
\end{tabular}


Tabela 3. Massa característica de metais em matriz de mel em $\mathrm{mg} \mathrm{kg}^{-1}$

\begin{tabular}{|c|c|c|c|c|c|c|c|c|}
\hline \multirow{2}{*}{ METAIS } & \multicolumn{8}{|c|}{ AMOSTRAS $\left(\mathrm{mg} \mathrm{kg}^{-1}\right)$} \\
\hline & $\mathrm{BC} 1$ & $\mathrm{BC} 2$ & $\mathrm{BC} 3$ & $\mathrm{BC} 4$ & BC5 & TU1 & TU2 & BA1 \\
\hline$\overline{\mathrm{Mn}}$ & $<\mathrm{LD}$ & $<\mathrm{LD}$ & $<\mathrm{LD}$ & $<\mathrm{LD}$ & $<\mathrm{LD}$ & $0,95 \pm 0,05$ & $<\mathrm{LD}$ & $1,3 \pm 0,05$ \\
\hline K & $335,8 \pm 39,8$ & $248,9 \pm 22,9$ & $74,56 \pm 5,7$ & $423,51 \pm 37$ & $462 \pm 23,1$ & $517 \pm 27,08$ & $29,1 \pm 1,3$ & $1594,7 \pm 67,7$ \\
\hline $\mathrm{Mg}$ & $18,3 \pm 1,8$ & $14,9 \pm 0,3$ & $<\mathrm{LD}$ & $11,8 \pm 1,05$ & $14,8 \pm 1,1$ & $35,0 \pm 2,8$ & $<\mathrm{LD}$ & $113,2 \pm 2,7$ \\
\hline $\mathrm{Fe}$ & $1,01 \pm 0,4$ & $0,97 \pm 0,6$ & $<\mathrm{LD}$ & $0,6 \pm 0,06$ & $2,73 \pm 0,8$ & $<\mathrm{LD}$ & $18,6 \pm 2,2$ & $0,7 \pm 0,65$ \\
\hline $\mathrm{Al}$ & $<\mathrm{LD}$ & $<\mathrm{LD}$ & $<\mathrm{LD}$ & $2 \pm 1,01$ & $8,8 \pm 6,1$ & $1,1 \pm 0,42$ & $1,1 \pm 0,3$ & $<\mathrm{LD}$ \\
\hline In & $<\mathrm{LD}$ & $<\mathrm{LD}$ & $2,4 \pm 0,2$ & $<\mathrm{LD}$ & $<\mathrm{LD}$ & $3,1 \pm 1,9$ & $2,9 \pm 1,4$ & $<\mathrm{LD}$ \\
\hline $\mathrm{Sr}$ & $<\mathrm{LD}$ & $<\mathrm{LD}$ & $<\mathrm{LD}$ & $<\mathrm{LD}$ & $<\mathrm{LD}$ & $<\mathrm{LD}$ & $<\mathrm{LD}$ & $0,7 \pm 0,07$ \\
\hline$\underline{B}$ & $3,5 \pm 0,7$ & $<\mathrm{LD}$ & $3,2 \pm 1,5$ & $5,4 \pm 0,4$ & $5 \pm 2,8$ & $3,4 \pm 1,2$ & $<\mathrm{LD}$ & $5,4 \pm 0,6$ \\
\hline \multirow{2}{*}{ METAIS } & \multicolumn{8}{|c|}{ AMOSTRAS $\left(\mathrm{mg} \mathrm{kg}^{-1}\right)$} \\
\hline & BA2 & BA3 & $\mathrm{SD}$ & $\mathrm{AR}$ & $\mathrm{SL}$ & SLZ1 & SLZ2 & \\
\hline$\overline{\mathrm{Ni}}$ & $<\mathrm{LD}$ & $<\mathrm{LD}$ & $<\mathrm{LD}$ & $<\mathrm{LD}$ & $<\mathrm{LD}$ & $<\mathrm{LD}$ & $0,9 \pm 0,09$ & \\
\hline $\mathrm{Pb}$ & $<\mathrm{LD}$ & $<\mathrm{LD}$ & $<\mathrm{LD}$ & $<\mathrm{LD}$ & $<\mathrm{LD}$ & $1,8 \pm 0,04$ & $3,0 \pm 0,08$ & \\
\hline $\mathrm{Cu}$ & $<\mathrm{LD}$ & $<\mathrm{LD}$ & $<\mathrm{LD}$ & $<\mathrm{LD}$ & $<\mathrm{LD}$ & $2,1 \pm 0,01$ & $3,7 \pm 0,2$ & \\
\hline $\mathrm{Mn}$ & $1,9 \pm 0,1$ & $<\mathrm{LD}$ & $<\mathrm{LD}$ & $2,7 \pm 0,1$ & $<\mathrm{LD}$ & $2,1 \pm 0,09$ & $1,25 \pm 0,1$ & \\
\hline $\mathrm{Zn}$ & $<\mathrm{LD}$ & $<\mathrm{LD}$ & $<\mathrm{LD}$ & $3,94 \pm 0,2$ & $<\mathrm{LD}$ & $1 \pm 0,1$ & $2 \pm 0,1$ & \\
\hline K & $578,2 \pm 28,8$ & $336,7 \pm 27,3$ & $264,9 \pm 5,3$ & $1085,2 \pm 75,2$ & $492,2 \pm 58,8$ & $1429,9 \pm 64,2$ & $1249,9 \pm 130,9$ & \\
\hline $\mathrm{Mg}$ & $15,8 \pm 1,4$ & $3,35 \pm 0,8$ & $<\mathrm{LD}$ & $23 \pm 2,2$ & $15,6 \pm 1,6$ & $44,8 \pm 1,9$ & $27,9 \pm 3,0$ & \\
\hline $\mathrm{Fe}$ & $1,4 \pm 0,05$ & $<\mathrm{LD}$ & $0,8 \pm 0,3$ & $2,3 \pm 0,25$ & $1,9 \pm 0,02$ & $2,9 \pm 0,3$ & $4,5 \pm 0,4$ & \\
\hline $\mathrm{Al}$ & $25,8 \pm 1,3$ & $3,2 \pm 0,9$ & $2,3 \pm 0,2$ & $5,3 \pm 3,0$ & $8,5 \pm 1,3$ & $10,5 \pm 0,8$ & $11,7 \pm 4,7$ & \\
\hline $\mathrm{Ga}$ & $<\mathrm{LD}$ & $<\mathrm{LD}$ & $<\mathrm{LD}$ & $<\mathrm{LD}$ & $<\mathrm{LD}$ & $8,1 \pm 0,2$ & $8,1 \pm 0,1$ & \\
\hline In & $<\mathrm{LD}$ & $3,6 \pm 2,5$ & $2,7 \pm 1,9$ & $3,6 \pm 1,6$ & $<\mathrm{LD}$ & $<\mathrm{LD}$ & $<\mathrm{LD}$ & \\
\hline$\underline{B}$ & $3,6 \pm 0,1$ & $<\mathrm{LD}$ & $<$ LD & $5,7 \pm 2,1$ & $5,8 \pm 2,3$ & $11,3 \pm 0,6$ & $9,3 \pm 3,2$ & \\
\hline
\end{tabular}

Resultados expressos como média \pm desvio padrão. <LD: menor que limite de detecção. BC - Bacabeira; TU - Tutóia; BA - Barreirinhas; SD - São Domingos; AR - Araguanã; SL - Santa Luzia; SLZ - São Luís.

extremamente prejudiciais à saúde, foram identificados nas amostras das áreas urbanas de São Luís. A massa característica de níquel no mel encontrada na amostra apresentou um limite tolerável, estando de acordo com o Decreto 55871/65 em que o máximo permitido é de $5,00 \mathrm{mg} \mathrm{kg}^{-1} \cdot{ }^{27}$ Duas amostras pertencentes a São Luís apresentaram chumbo em sua composição, em níveis consideravelmente elevados, que ultrapassaram o limite de $0,30 \mathrm{mg} \mathrm{kg}^{-1}$ estabelecido pela Instrução Normativa $\mathrm{N}^{\circ}$ 88/21 (RN 487/21). ${ }^{28}$ Valores parecidos também foram relatados em estudos com matriz de mel realizados por Yucel e Sultanoglu, ${ }^{25}$ com concentrações que variaram entre 0,06-2,02 $\mathrm{mg} \mathrm{kg}^{-1}$ e Oliveira e Nagashima ${ }^{22}$ reportando valores entre 0,03-4,08 $\mathrm{mg} \mathrm{kg}^{-1}$ para o chumbo. ${ }^{22,25}$ Por ser um metal tóxico é importante o frequente monitoramento em relação à presença desses componentes já que o mel é uma substância ácida que favorece a absorção de metais. ${ }^{29}$ Nesse estudo, a presença de chumbo no mel está fortemente associada ao local de produção estar próximo a ambientes inóspitos como aterros sanitários e as possíveis práticas inadequadas de apicultura.

Em relação ao cobre, considerado como um elemento traço essencial, o limite tolerável pela legislação para este componente no mel é $10,00 \mathrm{mg} \mathrm{kg}^{-1}$, também de acordo com a Instrução Normativa $\mathrm{N}^{\circ} 88 / 21$ (RN 487/21). Nas amostras analisadas, o cobre variou entre 2,1-3,7 mg kg-1. ${ }^{28}$ Estudos feitos pelos autores citados também relataram valores semelhantes desses elementos no mel. A presença desses componentes pode ser explicada em razão da localização onde foram recolhidas as amostras, pois são áreas próximas a setores industriais como portos e mineradoras. Essas instalações possibilitam a fácil contaminação de solos e lençóis freáticos próximos as colmeias por esses elementos. ${ }^{30}$

A diversidade de origem botânica e geográfica são os principais fatores responsáveis pela variação de concentração dos elementos presentes nos méis produzidos no Maranhão. O alumínio variou em uma faixa de massa entre $1,1-25,8 \mathrm{mg} \mathrm{kg}^{-1}$, sendo que os valores mais altos foram encontrados no município de Barreirinhas, Bacabeira,
Santa Luzia e na área urbana de São Luís. Este metal está presente no meio ambiente, por atividades antropogênicas, como descartes de resíduos industriais, mineração e produção agrícola, justificando assim a concentração encontrada no mel. Além disso, o mel pode ser contaminado durante seu processamento, através dos equipamentos e ferramentas utilizadas. Materiais como alumínio, aço inoxidável e aço galvanizado usados em ferramentas e equipamentos para o processamento do mel podem liberar alguns metais poluentes (incluindo $\mathrm{Al}, \mathrm{Cd}, \mathrm{Co}, \mathrm{Cr}, \mathrm{Cu}, \mathrm{Fe}, \mathrm{Pb}, \mathrm{Ni}$ e $\mathrm{Zn}$ ). ${ }^{31}$ Esses valores estão em concordância com um estudo realizado por Tutun e colaboradores, com amostras de mel analisados na Turquia. ${ }^{32}$

Apesar de não serem comumente evidenciados em estudos com mel, o gálio $\left(8,1 \mathrm{mg} \mathrm{kg}^{-1}\right)$, boro $\left(3,2-11,3 \mathrm{mg} \mathrm{kg}^{-1}\right)$ e estrôncio $\left(0,7 \mathrm{mg} \mathrm{kg}^{-1}\right)$ também foram identificados nas amostras analisadas. $\mathrm{O}$ Ga é normalmente encontrado na forma de hidróxidos e como subproduto em processos de obtenção de outros metais. ${ }^{33}$ Já o B, pode ser resultante do uso de fertilizantes e inseticidas em solos e plantas, para o controle de insetos em áreas próximas ao setor de produção do mel. Ainda não há limites estabelecidos para esses elementos em mel, porém o consumo excessivo pode acarretar efeitos colaterais como náuseas e diarreia. ${ }^{34-35}$ Valores parecidos para o boro foram reportados por Chudzinska e Baralkiewicz. ${ }^{20}$

\section{Tratamento quimiométrico de dados}

Com o emprego de ferramentas estatísticas, foi possível observar uma correlação entre as variáveis e as amostras de mel por meio dos dados obtidos após a determinação dos elementos inorgânicos. Esses dados foram submetidos à Análise de Componentes Principais (PCA) e Análise Hierárquica de Clusters (HCA).

Para que todas as variáveis tivessem influências equitativas na distribuição dos resultados, os dados originais foram pré-processados através do auto escalonamento em razão das concentrações dos metais apresentarem diferentes ordens de magnitude. O Ké um elemento que 
possui grande impacto devido sua alta concentração e, dessa forma, pode acabar dominando completamente a organização dos resultados, devido seu grande efeito na medida das distâncias, resultando em tendências diferentes. Assim, o pré-processamento garante o mesmo grau de importância para todas as variáveis, convertendo os dados a escalas comparáveis.

Na Figura 2, os dados auto-escalonados foram submetidos à análise de PCA, sendo possível observar a formação de duas componentes principais. A PC1 respondeu por $44 \%$ da discriminação, ficando clara sua relação com atividades poluidoras. No gráfico de scores (Figura $2 \mathrm{~A}$ ), quando analisado sob a perspectiva da primeira componente (PC1), é possível observar uma nítida separação entre as amostras de mel obtidas em São Luís e as demais amostras do interior do estado. Essa separação pode ser explicada através do gráfico de loadings (Figura 2 B), observando-se a separação na primeira componente que está fortemente associada aos valores de $\mathrm{Cu}, \mathrm{Pb}, \mathrm{Ni}, \mathrm{Ga}$ e $\mathrm{B}$, enquanto que na $\mathrm{PC} 2$ as variáveis dominantes são $\mathrm{Mn}, \mathrm{Zn}, \mathrm{Al}, \mathrm{K}$ e $\mathrm{Mg}$.

Os gráficos permitem observar que a $\mathrm{PC} 1$ tem relação principalmente com a poluição ambiental nos arredores das colmeias, enquanto que a PC2 se associa a características de solo e práticas de agricultura. A variação das concentrações dos elementos encontrados no mel pode ser explicada por uma série de fatores antropogênicos como práticas de agricultura, tráfego de automóveis e atividades industriais nas proximidades das colmeias. ${ }^{36}$

Percebe-se que as amostras de São Luís, estão mais distintas quando comparadas as outras amostras e, isso se deve ao fato desse ambiente em específico estar rodeado por uma região com intensa atividade industrial e degradação ambiental, o que influencia diretamente na presença de traços de metais.

$\mathrm{O} \mathrm{Pb}$, elemento tóxico encontrado com alto teor, pode estar associado a proximidade do apiário a um aterro sanitário desativado. Estudos feitos por Campos e colaboradores relataram a presença de chumbo acima dos níveis estabelecidos pela legislação brasileira nas águas de um Igarapé localizado nas proximidades do aterro. Vale destacar ainda que essa região é caracterizada por atividades agrícolas como a produção de hortaliças e frutíferas, o que provavelmente contribui como fonte de elementos tóxicos pelo uso de fertilizantes. ${ }^{37}$

Ainda de acordo com a Figura 2, pode-se observar que duas amostras do município de Barrerinhas (BA), por apresentarem semelhanças quanto ao teor de metal, encontram-se agrupadas enquanto que uma terceira amostra do mesmo município encontrase junto às demais amostras do interior. As amostras de BA que apresentam similaridade foram obtidas na mesma localidade.

Similaridades dentro do grupo do interior também foram observadas entre as amostras de Bacabeira (BC), uma vez que formam um subgrupo dentro do grupo do interior.

Essas informações observadas por meio das análises de componentes principais foram confirmadas através do dendrograma obtido pela HCA (Figura 3). A análise de agrupamento foi aplicada para os dados pré-processados e a distância euclidiana foi utilizada para calcular as similaridades entre as amostras. $\mathrm{O}$ método permitiu exibir uma tendência em classificar diferentes classes de mel de acordo com seu perfil mineral. Neste gráfico observa-se a formação
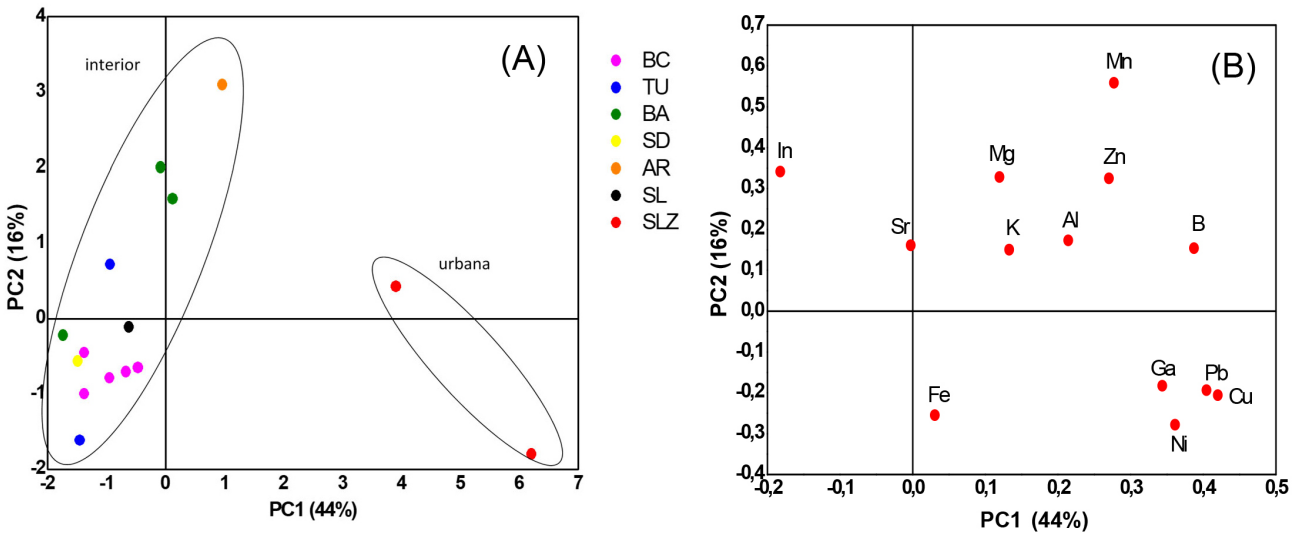

Figura 2. (A)Gráfico de scores de PC1 versus PC2 das concentrações de metais nas amostras de mel. BC - Bacabeira; TU - Tutóia; BA - Barreirinhas; SD São Domingos; AR - Araguanã; SL - Santa Luzia; SLZ - São Luís. (B) Gráfico de Loadings (PC1 vs PC2) aplicado às variáveis

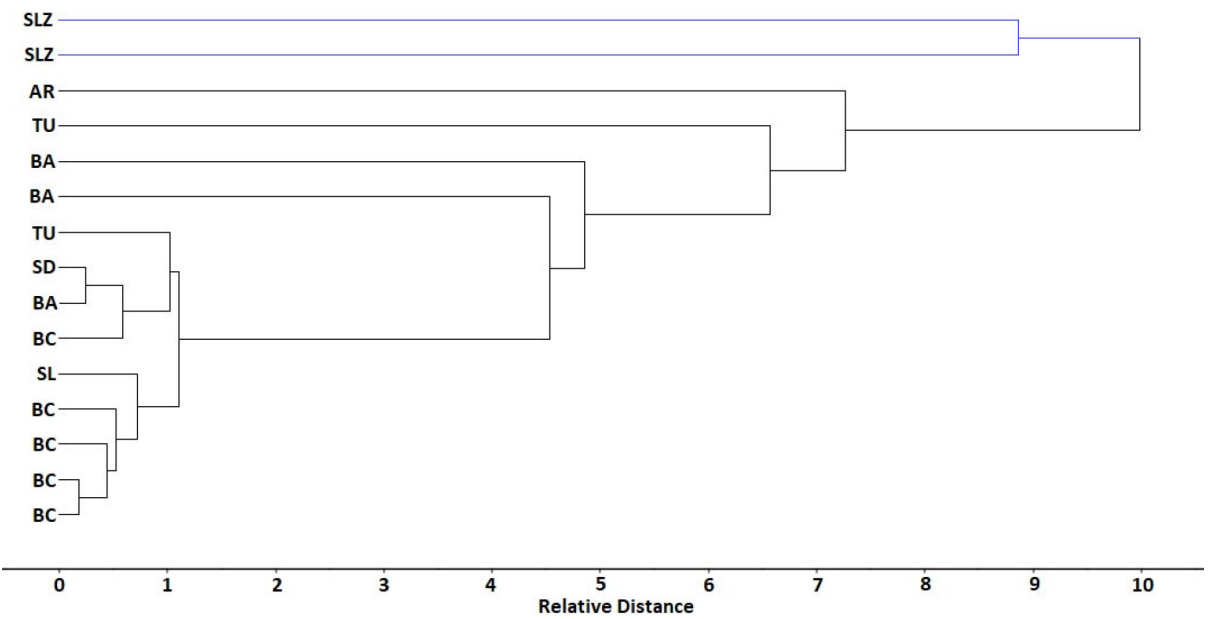

Figura 3. Dendrograma de HCA de amostras de mel de diferentes regiões do Maranhão. SLZ - São Luís; AR - Araguanã; TU - Tutóia; BA - Barreirinhas; $S D$ - São Domingos; BC-Bacabeira; SL - Santa Luzia. 
de dois agrupamentos principais: urbana (linha azul) e interior (linha preta). No agrupamento urbano, as amostras SLZ são equidistantes entre si, significando que as variáveis determinadas possuem altos níveis de similaridade. Por outro lado, essas amostras apresentamse bem diferentes das demais. Em relação aos grupos de amostras do interior, observou-se a subdivisão em vários subgrupos, sendo possível identificar uma alta similaridade entre as amostras de Bacabeira (BC), assim como entre Barreirinhas (BA) e São Domingos (SD). Esse comportamento sugere uma correlação entre o local de origem das amostras e as variáveis estudadas. Entretanto, destaca-se a necessidade de um conjunto maior de amostras para confirmar a tendência apresentada.

\section{CONCLUSÃO}

No presente estudo, 15 amostras de mel obtidas de produtores rurais do estado do Maranhão foram caracterizadas de acordo com seu conteúdo mineral. As massas características de 19 elementos foram determinadas por ICP OES, apresentando uma ampla faixa de concentração, e em particular altos níveis de $\mathrm{K}$ e Mg. Os resultados mostraram ainda que amostras obtidas na área urbana (industrializada), apresentaram maiores quantidades de elementos, inclusive metais tóxicos como $\mathrm{Pb}$ e $\mathrm{Ni}$. Os resultados encontrados foram compatíveis com outros estudos da literatura, porém a concentração de $\mathrm{Pb}$ na área urbana encontrava-se acima do valor estabelecido na legislação. Métodos quimiométricos, como PCA e HCA foram utilizados no estudo de classificação e agrupamento das amostras de mel, sendo possível observar uma separação bem definida em dois grupos principais: amostras urbanas e rurais. Esses resultados embora preliminares confirmam que o mel pode ser utilizado tanto como bioindicador de origem geográfica quanto de contaminação ambiental, demonstrando a importância do monitoramento dos níveis de elementos inorgânicos em mel, como forma de garantir a saúde pública e integridade do produto. Destaca-se ainda que um número maior de amostras seria necessário para melhor correlacionar o teor mineral com o local de origem, especialmente com relação ao $\mathrm{Pb}$, metal tóxico encontrado em níveis elevados.

\section{AGRADECIMENTOS}

O presente trabalho foi realizado com o apoio da Fundação de Amparo à Pesquisa e ao Desenvolvimento Científico e Tecnológico do Maranhão - FAPEMA. Os autores também agradecem ao Programa de Pós-Graduação em Química (PPGQ/IFMA) e aos professores responsáveis pelo Laboratório de Análise e Pesquisa de Alimentos, Bebidas e Ambiental - LAPAA, que disponibilizaram o ambiente para o desenvolvimento da pesquisa.

\section{REFERÊNCIAS}

1. Bilandžić, N.; Đokić, M.; Sedak, M.; Kolanović, B. S.; Varenina, I.; Končurat, A.; Rudan, N. Food Chemistry, 2011, 128, 4.

2. Pohl, P.; Sergiel, I. Microchimica Acta, 2010, 168, 1.

3. Dourado, G. S.; Gomes, V. V.; Maia, M. T. V.; Vasconcelos, A. A.; Costa, K. S.; Faial, K. C.; Carneiro, B. S.; Vasconcelos Junior, N. T.; Taube, P. S. Br. J. Anal. Chem, 2019, 6, 23.

4. Abu-Tarboush, H. M.; Al-Kahtani, H. A.; El-Sarrage, M. S. Food Chemistry, 1993, 46, 1.

5. Stankovska, E.; Stafilov, T.; Saljn, R. Environment Acess, 2008, 142, 1.

6. Anklam, E. Food Chem. 1998, 63, 4.

7. Oliveira, F. A; Tese de Doutorado, Universidade Federal de Ouro Preto, Brasil, 2017.
8. Yucel, Y.; Sultanoglu, P. Food Chem, 2013, 140, 1.

9. Sousa, R. A.; Neto, W. B.; Poppi, R. J.; Baccam, N.; Cadore, S. Quim. Nova, 2006, 29, 4.

10. Silveira-Júnior, C. E. A.; Silva, G. R.; Gomes, A. N. P.; Silva, T. M. S.; Arruda, R.; Santos, F. A. R. Journal of Apicultural Research, 2020, 0021-8839.

11. Dhahir, S. A.; Hemed, A. H. American Journal of Applied Chemistry, 2015, 3,3 .

12. Hungerford, N. L.; Tinggi, U.; LL Tan, B.; Farrell, M.; Fletcher, M. T. Int J Environ Res Saúde Pública, 2020, 17, 17.

13. Velimirović, D.; Tošić, S.; Mitić, S.; Pavlović, A.; Rašić, I.; Stojanović, M \& G. Journal of Apictural Research, (2021). doi.org/10.1080/00218 839.2021.1898783.

14. Leme, A.B.P.; Bianchi, S.R.; Carneiro, R.L.; Nogueira, A. R. A. Food Anal. Methods, (2014). doi.org/10.1007/s12161-013-9706-5.

15. Ioannidou, M. D.; Zachariadis, G. A.; Anthemidis, A. N.; Stratis, J. A. Talanta, 2005, 65, 1.

16. Di Bella, G.; Lo Turco, Vicenzo.; Portoti, A. G.; Bua, G. D.; Fede, M. R.; Dugo, G. Journal of Food Composition and Analysis, 2015, 44, 25.

17. Currie, L. A. Analytica Chimica Acta 1999, 391, 2.

18. Esbensen, K.; Swarbrick, B.; Westad, F. CAMO Software AS, 2017, p. 107.

19. Conti, M. E.; Canepari, S.; Finoia, M. G.; Mele, G.; Astolfi, M. L. Journal of Food Composition and Analysis, 2018, 74, 102.

20. Chudzinska, M. Baralkiewicz, D. Food and Chemical Toxicology, 2010, 48,1 .

21. Mendes, T. M. F. F.; Bacan, S.N.; Cadores, S. J. Braz. Chem. Soc, 2006, $17,1$.

22. Oliveira, K. M. G.; Nagashima, L. A. Ambiência Guarapiava, 2018, 14, 1.

23. Zaric, N. M.; Ilijevic, K.; Stanisavljevic, L. \& Grzetic, I. International Journal of Environmental Science and Technology, 2017, 13, 2.

24. POHL, P. Trends Anal, 2009, $28,1$.

25. Yucel, Y.; Sultanoglu, P. Food Chemistry, 2012, 135, 1.

26. Squadrone, S.; Brizio, P.; Stella, C.; Mantia, M.; Pederiva, S.; Brusa, F.; Mogliotti, P.; Garrone, A.; Cesarina, A. M. Environmental Science and Pollution Research Springer-Verlag GmbH Germany, part of Springer Nature, 2020, 27, 11.

27. BRASIL. Ministério da Saúde. Decreto no 55.871/1965. Modifica o Decreto $n$ 50.040/1961, referente às normas reguladoras do emprego de aditivos para alimentos. Diário oficial da União, Brasília, DF, 9 de abril. 1965.

28. ANVISA. Instrução Normativa - IN N ${ }^{\circ} 88$ (RN 487). Diário Oficial da União, Brasília, DF, 26 de março. 2021.

29. Karabagias, I. K. Acta Scientific Nutritional Health, 2017, 15.

30. Silva, C. A.; Alencar, M. H. B. C. Sau. \& Transf. Soc., 2014, 5, 1.

31. Solayman, Md.; Asiful Islam, Md., Sudip, P.; Yousuf, A.; Khalil, Md. I.; Alam, N. and Gan, S. H. Institute of Food Technologists., 2016, 15, 1.

32. Tutun, H.; Ahu Kahraman, H.; Aluc, Y.; Avci, T.; Ekici, H. Vet Res Forum 2019; 10, 3.

33. Kramer, D. A. Gallium and gallium arsenide: Supply, Technology and Uses. University of Michigan Library,1988.

34. Siede, R.; Dyrba, W.; Augustin, T., Wiegand, A., \& Ellinghaus, R. Journal of Applied Entomology, 2013, 137,9 .

35. Valdovino-Flores, C.; Gaspar-Ramirez, O.; Heras-Ramirez, M. E.; LaraÁlvarez, C.; Dorantes-Ugalde, J. A.; Saldana-Loza, L. M. Plos One, 2016, $11,4$.

36. Vincevica-Gaile, Z.; Klavins, M.; Rudovica, V. \& Viksna, A. Transactions on Ecology and The Environment, 2011, 167.

37. Campos, A. E. L.; Nunes, G. S.; Oliveira, J. C. S.; Toscano, I. A. S. Quím. Nova, 2009, 32, 4. 